\title{
THE
}

1996

\section{Cloud Detection from a Sequence of SST Images}

Jean-François Cayula

University of Rhode Island

Peter C. Cornillon

University of Rhode Island, pcornillon@uri.edu

Follow this and additional works at: https://digitalcommons.uri.edu/gsofacpubs

Terms of Use

All rights reserved under copyright.

\section{Citation/Publisher Attribution}

Cayula, J-F., \& Cornillon, P. (1996). Cloud Detection from a Sequence of SST Images. Remote Sensing of Environment, 55(1), 80-88.

Available at: http://dx.doi.org/10.1016/0034-4257(95)00199-9

This Article is brought to you for free and open access by the Graduate School of Oceanography at DigitalCommons@URI. It has been accepted for inclusion in Graduate School of Oceanography Faculty Publications by an authorized administrator of DigitalCommons@URI. For more information, please contact digitalcommons-group@uri.edu. 


\title{
Cloud Detection from a Sequence of SST Images
}

\author{
Jean-François Cayula ${ }^{* \dagger}$ and Peter Cornillon ${ }^{*}$
}

\begin{abstract}
A to a companion edge-detection algorithm. The cloud detection integrates two distinct algorithms: one based on multiimage processing, the other on single-image analysis. The multiimage portion of the cloud detection algorithm operates on a time sequence of sea surface temperature (SST) images. It is designed to detect clouds associated with regions of apparently lower temperatures than the underlying SST field. A pixel in the current image is initially considered to be corrupted by clouds if it is significantly cooler than the corresponding pixel in a neighbor image. To refine the initial classification, the algorithm checks the current image and the neighbor image for the presence of water masses, which through displacement could explain the change in temperature. The single-image cloud detection algorithm is designed to detect clouds associated with regions of the SST image where gradient vectors have a large magnitude. These regions are flagged in the map of potential clouds. Multiimage processing is integrated with the single-image algorithm by adding pixels classified as cloudy at the multiimage level to the map of potential clouds. Further analysis of the gradient vector field and of the shapes of potentially cloudy areas allows one to determine whether these regions correspond to clouds or SST fronts. A previous study has shown that the clouds identified by the single-image algorithm were in close agreement with those detected by a human expert. To validate the additional multiimage processing, the effect of the integrated cloud detection on the performance of a companion edge detection algorithm is examined. These results and a direct comparison with the cloud masks produced by a human expert indicate
\end{abstract}

\footnotetext{
${ }^{*}$ Graduate School of Oceanography, University of Rhode Island, Narragansett

${ }^{\dagger}$ Planning Systems Inc., Stennis Space Center, Mississippi

Address correspondence to Jean-François Cayula, Planning Systems, Inc., Bldg. 9121, MSAAP, Stennis Space Center, MS 39529. E mail: j.cayula@ieee.org

Received 21 March 1994; revised 5 August 1995.
}

that, compared to the single-image algorithm, the multiimage algorithm successfully identify additional cloudcorrupted regions while keeping a low rate for the detection of false clouds.

\section{INTRODUCTION}

Satellite remote sensing of the oceans offers almost continuous worldwide coverage not possible to achieve from in situ observations. As a result, satellites have become a major source of information for the study of oceanographic processes. For example, oceanographers commonly use SST images, produced by the satelliteborne Advanced Very High Resolution Radiometers (AVHRRs), to locate features and to study the dynamic behavior of the oceans. Computer processing of SST images with as little human intervention as possible has become increasingly popular because of the abundance and the electronic nature of the data (Podestá et al., 1993).

Clouds are a major cause of erroneous temperature observations (Stewart, 1985). In the context of edge detection, the analysis of SST fields in which clouds are left undetected is likely to produce false fronts and thus make the edge detection process unreliable. Therefore, the successful processing and analysis of SST fields must include the accurate identification of regions that are contaminated by clouds.

Although other AVHRR channels can help determine cloudiness (Derrien et al., 1990; Gallegos et al., 1993), the present algorithm concentrates on cloud detection through the analysis of satellite-derived SST fields. As a result, the cloud detection algorithm can operate on the same (readily available) data as those used by edge detection algorithms (Cayula and Cornillon, 1995). The cloud detection algorithm integrates two methods: multiimage cloud detection and single-image cloud detection. Figure 1 shows the flowchart of the algorithm.

The purpose of the multiimage algorithm (next sec- 


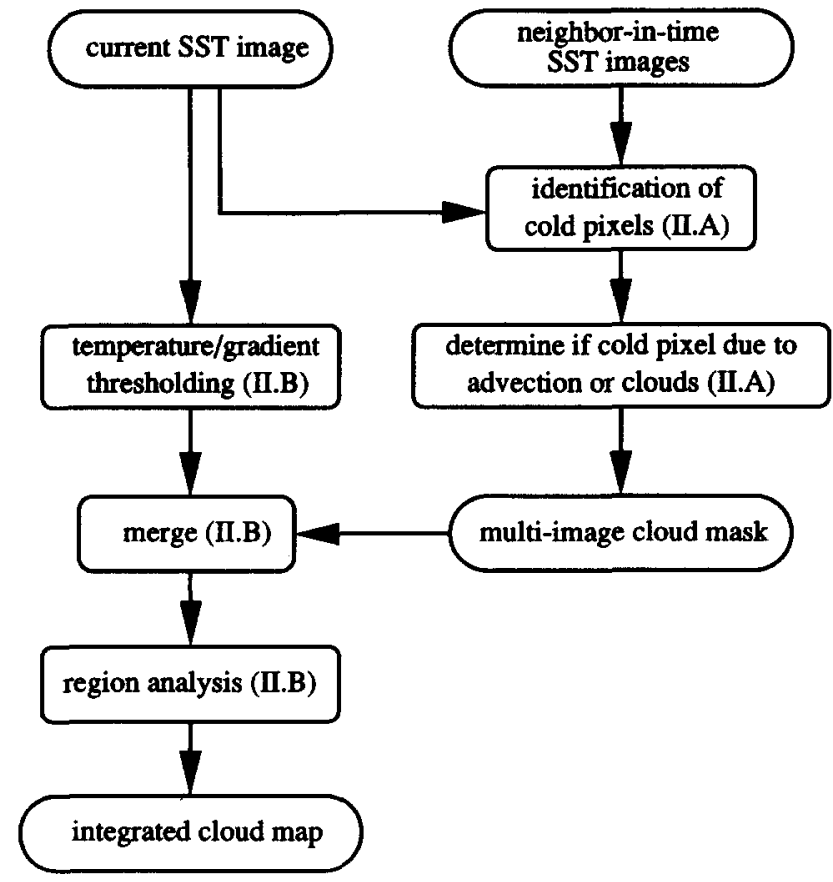

Figure 1. Flow chart of the algorithm indicating the section in which a particular step is explained.

tion) is to detect clouds associated with regions of apparently cooler temperature than the underlying SST field. To achieve this task, the algorithm analyses a time sequence of images. First, pixels in one image (the current image) that are significantly cooler than their counterparts in images adjacent to the current image in the sequence (neighbor images) are flagged as cold pixels. Because clouds are usually less persistent than SST features (Stewart, 1985), it is possible to differentiate between relatively cooler pixels resulting from the displacement of SST features and those resulting from clouds. For each image in the time sequence, a cold pixel in the current image is classified as potentially cloudy if a similarly cold water mass cannot be found near the corresponding location in the neighbor image.

The single-image cloud detection algorithm (next section) adds to the map of potentially cloudy pixels identified in the multiimage pass those pixels with a gradient magnitude greater than a predefined threshold. Connected regions in the map of potential clouds are then analyzed individually to refine the preliminary classification. The algorithm uses the shape of a connected region and the gradient vector field within a connected region to classify the region.

In a series of test images, Kowalski et al. (1991) demonstrated that a single-image cloud detection algorithm, similar to the one described in the previous paragraph without the benefit of the multiimage pass, produced results in close agreement with those of a trained analyst. However, clouds left undetected by the single-image cloud detection algorithm can still significantly affect the quality of tasks such as automated edge detection. Use of the integrated cloud detection algorithm corrects this problem so that automatically detected SST fronts compare well with those identified by a human expert (the third section).

The satellite data were obtained from the Advanced Very High Resolution Radiometer (AVHRR) of the TIROS-N series satellites (Schwalb, 1978). Following navigation, the data were remapped to a common projection. The SST field was calculated using the twochannel algorithm of McClain et al. (1983) which operates on Channels 4 and $5(10.5-11.3 \mu \mathrm{m}$ and 11.5$12.5 \mu \mathrm{m})$. For all the images, image intensity, $i_{x, y}$ and temperature were related by

$$
i_{x, y}=8 \cdot \tau_{x, y}
$$

where $\tau_{x, y}$ represents the SST value expressed in ${ }^{\circ} \mathrm{C}$ at location $(x, y)$ and $i_{x, y}$ is the corresponding image intensity. Temperatures higher than $32^{\circ} \mathrm{C}$ were set to an image intensity of 255 while temperatures lower than $0.5^{\circ} \mathrm{C}$ were thresholded to 0 . Two sets of images were available for this study. The 300 images in the first set had a spatial resolution of $2 \mathrm{~km} /$ pixel and covered an area from $33^{\circ} \mathrm{N}$ to $44^{\circ} \mathrm{N}$ and $76^{\circ} \mathrm{W}$ to $63^{\circ} \mathrm{W}$. A subset of this group of images was used to develop the algorithm. The 98 images in the second set had a spatial resolution of $1 \mathrm{~km} /$ pixel and covered an area of from $34^{\circ} \mathrm{N}$ to $39^{\circ} \mathrm{N}$ and $76^{\circ} \mathrm{W}$ to $69^{\circ} \mathrm{W}$. A more extensive discussion of the processing steps involved in generating these two data sets may be found in Cornillon et al. (1987).

\section{THE ALGORITHM}

\section{Multiimage Analysis}

Cloud contaminated pixels in satellite-derived SST fields are generally colder than unobscured pixels (Steward, 1985). This section presents a method designed to identify the colder-than-expected cloud-contaminated pixels. The method is based on the comparative analysis of a time sequence of SST images where all images in the sequence cover the exact same area. In this context, the current image is defined as the image in which clouds are to be identified. Neighbor images are the images in the sequence that occur within $50 \mathrm{~h}$ (before or after) of the current image. Although longer intervals of time can be used for some regions of the ocean, the dynamic nature of the Gulf Stream region requires intervals shorter than $\pm 50 \mathrm{~h}$ to produce useful information (results slowly degrade for longer intervals).

The algorithm operates on one neighbor image at a time until all the existing neighbor images have been used. The coordinates $(x, y)^{c}$ and $(x, y)^{n}$ refer to the pixels at location $(x, y)$, respectively, in the current image and 
in the neighbor image. The " $c$ " superscript for "current" and " $n$ " superscript for "neighbor" are used throughout the text; e.g., the value of the SST field at $(x, y)^{c}$ is $\tau_{x, y}^{c}$ while that at $(x, y)^{n}$ is $\tau_{x, y}^{n}$.

The algorithm includes a processing step to deal with temperature offsets between images. This processing step, which is presented in Cayula (1993), had to be applied to our images because of poor temperature calibration. Retrieval algorithms making use of the improved Pathfinder calibration for AVHRR data, such as the Pathfinder SST algorithm, do not appear to require this correction.

\section{Preliminary Cloud Detection}

The actual data analysis starts with the identification of cold pixels in the current image. This step consists of a simple comparison between the SST value of a pixel in the current image and that of the corresponding pixel in the neighbor image. The pixel in the current image is considered to be a cold pixel if it is colder than the corresponding pixel in the neighbor image by $2.5^{\circ} \mathrm{C}$ :

$$
\tau_{x, y}^{n}-\tau_{x, y}^{c}>2.5^{\circ} \mathrm{C} \Rightarrow(x, y)^{c} \text { is a cold pixel. }
$$

This threshold was selected to avoid the effect of temperature offset between images and diurnal warming events. Although this is one of the most sensitive parameters of the algorithm, a change of $\pm 20 \%$ for this threshold produces less than a $\mp 3 \%$ change in number of cloudclassified pixels and agreement with the analyst (see validation in the next section) varies by less than $\pm 1 \%$.

\section{Refined Cloud Detection}

If the pixel $(x, y)^{c}$ is cold [Eq. (2)], then further analysis determines whether $(x, y)^{c}$ is cloudy. Classification of the cold pixel $(x, y)^{c}$ as cloudy relies on the analysis of the SST field that surrounds the location $(x, y)$ in the current image and the neighbor image. The area to be analyzed, $\mathscr{W}_{x, y}$, is a $\delta \mathrm{km}$ by $\delta \mathrm{km}$ window centered on $(x, y)$. If $\delta$ is appropriately chosen, based on the expected displacement of SST features, then it is possible to distinguish between relatively cooler pixels resulting from clouds and those resulting from such SST phenomena as the advection of oceanographic fronts. This distinction is possible because SST features usually do not move as fast as clouds (Cushman-Roisin, 1994).

In the Gulf Stream region off Cape Hatteras, with most fronts moving at less than $20 \mathrm{~km}$ per day (Cayula and Cornillon, 1995), a value of about $50 \mathrm{~km}$ seems appropriate for $\delta$. Considerations about the algorithm implementation, which requires $\delta=4 \cdot(2 k+1) \mathrm{km}$ where $k$ is an integer, lead to $\delta=44 \mathrm{~km}$. Choosing a higher value yields only marginal improvements: With $\delta=60 \mathrm{~km}$, the rate of agreement with the analyst (see next section) increases by less than $0.1 \%$ while processing time almost doubles. (Because the images that we have used to test the algorithm include the Gulf Stream, one of the strongest currents in the world ocean, hence a region that will include among the most rapidly

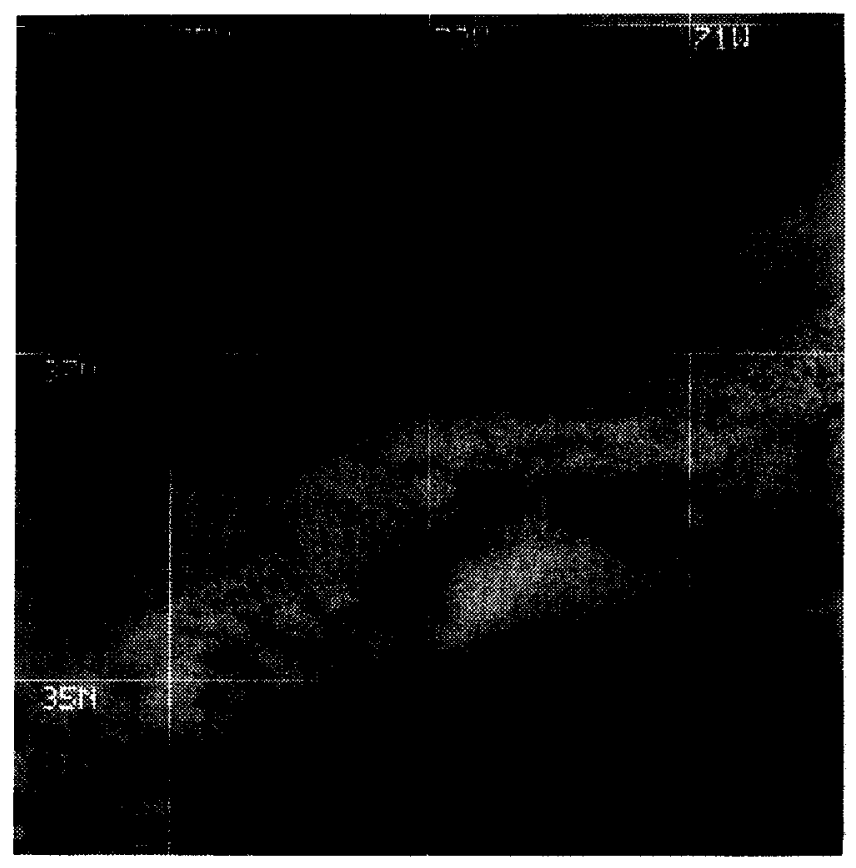

Figure 2. Image of SST field taken on 16 April 1982 at 7: 16 local time. Longitude and latitude coordinates are overlaid in white.

translating oceanographic features, we expect this value of $44 \mathrm{~km}$ to hold in other regions of the world.) The current implementation of the algorithm samples the windows $\mathscr{W}_{x, y}$ to speed up the computation so that only 1 pixel every $4 \mathrm{~km}$ is used. For images with $1 \mathrm{~km}$ resolution this means that a $44 \mathrm{~km}$ by $44 \mathrm{~km}$ region corresponds to an 11 by 11 pixel window.

If an SST front causes the difference between $\tau_{x, y}^{c}$ and $\tau_{x, y}^{n}$, then a mass of warm water approximately equal to $\tau_{x, y}^{n}$ and a mass of cold water with a temperature approximately equal to $\tau_{x, y}^{c}$ should both be found within $\mathscr{W}_{x, y}$. However, if the temperature difference is due to a cloud in that region of the current image, then the cloud is likely to have disappeared from that region in the neighbor image, and one should not expect to find a cold area with a temperature of $\tau_{x, y}^{c}$ within $\mathscr{W}_{x, y}$ in the neighbor image. To summarize,

$$
\left\{\begin{array}{c}
(x, y)^{c} \text { is a cold pixel }(2) \\
+ \\
\text { warm water mass exists within } \\
\mathscr{W}_{x, y} \text { in neighbor image } \\
+ \\
\text { cold water mass does not exist within } \\
\mathscr{W}_{x, y} \text { in neighbor image }
\end{array}\right\} \Rightarrow(x, y)^{c} \in \mathscr{C}_{p c}
$$

where $\mathscr{C}_{p c}$ is the map of potentially cloudy pixels. Figures 2 and 3 show two images from the sequence. The first image, one of the neighbor in time, depicts the satellitederived SST fields on 16 April 1982 while the second one on 17 April 1982 is the current image. Figure 4 represents, overlaid on Figures 3 , the map of potentially cloudy pixels that results from the multiimage step. 


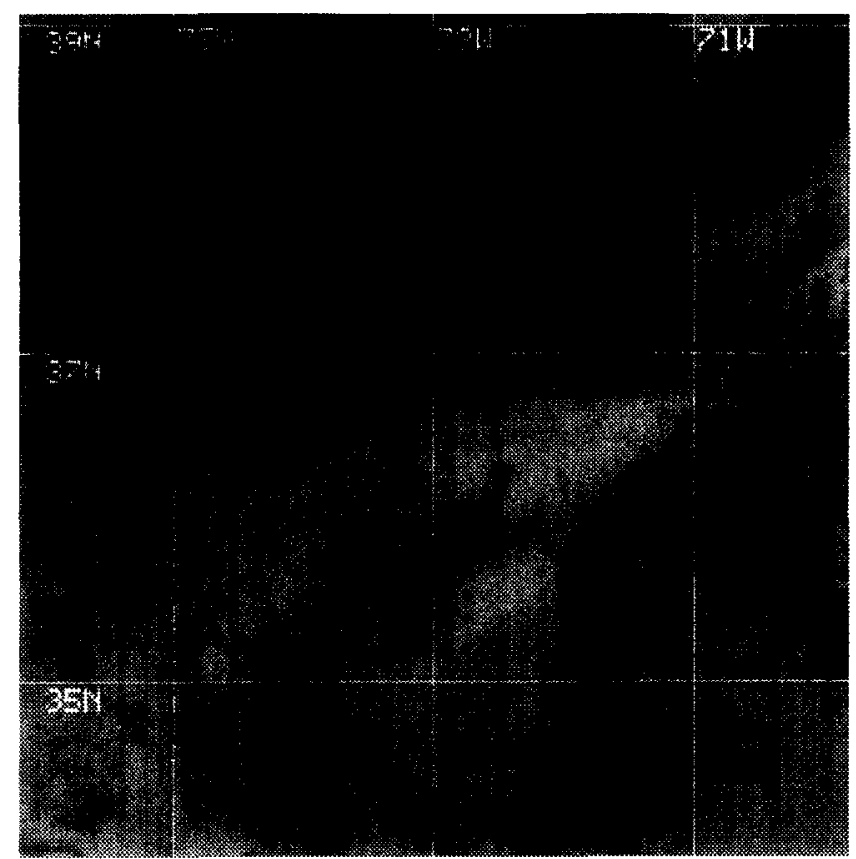

Figure 3. Image of the SST field taken on 17 April 1982 at 7:04 local time. Longitude and latitude coordinates are overlaid in white.

The next two subsections describe the criteria used to determine the presence of warm and cold water masses.

\section{Mass of Warm Water}

The algorithm verifies that $(x, y)^{n}$, the pixel at location $(x, y)$ in the neighbor image, is not the only warm pixel in the region, that is, that a mass of warm water exists, by counting the number of surrounding pixels $(i, j)^{n}$ which are warm ("\#" stands for "number of pixels in the set"),

$$
N_{x, y}^{\mathrm{warm}}=\#\left\{(i, j)^{n} \in \mathscr{H}_{x, y} \text { such that } \tau_{i, j}^{n}>\left(\tau_{x, y}^{n}-0.5^{\circ} \mathrm{C}\right)\right\} .
$$

The previous equation includes a $0.5^{\circ} \mathrm{C}$ tolerance which reflects the expected accuracy of the data (Cornillon and Straumma, 1985). The mass of warm water is said to exist in the neighbor image if

$$
N_{x, y}^{\text {warm }}>\varepsilon_{w}
$$

where $\varepsilon_{w}$ is an empirically determined threshold. In the current implementation, using an 11 by 11 pixel window, $\varepsilon_{w}$ is set to 5 . Any $\varepsilon_{w} \in[3,10]$ produces similar results on the data sets.

\section{Mass of Cold Water}

The next step determines the presence or absence, in the neighbor image, of a mass of cold water that can explain the difference of temperature between $(x, y)^{c}$ and $(x, y)^{n}$.

To reduce the amount of computation, we first check that $\tau_{x, y}^{c}$ is a valid SST value. We found by experimenting with various values that $\tau_{x, y}^{c}$ is unlikely to repre-

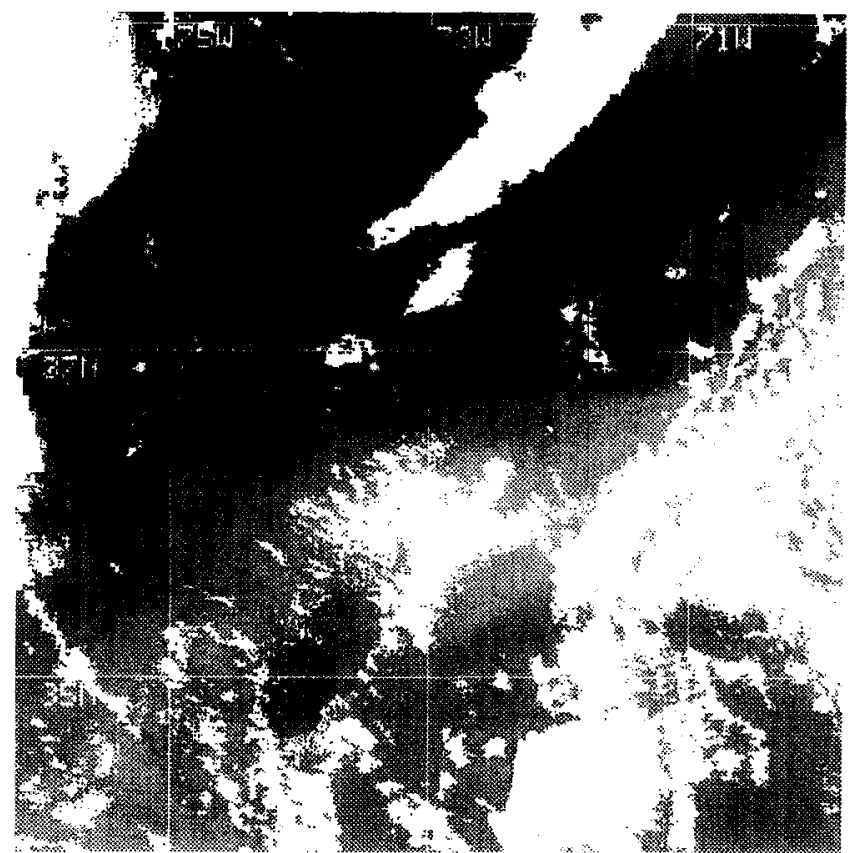

Figure 4. Image of the SST field shown in Figure 3. The map of potentially cloudy pixels that results from the multiimage step, as well as longitude and latitude coordinates, are overlaid in white.

sent a valid SST value if it is more than $18^{\circ} \mathrm{C}$ colder than $\tau_{x, y}^{n}$. This threshold is quite conservative in that, to the best of our knowledge, an $18^{\circ} \mathrm{C}$ temperature step, or larger, in $60 \mathrm{~km}$ has never been observed in the world ocean. In other regions of the oceans, the threshold could be substantially decreased because the temperature differences in those regions are usually smaller than in the Gulf Stream region.

The determination of whether a cold water mass exists is similar to that of the warm water mass and relies on a count of all valid cold pixels within the window $\mathscr{W}_{x, y}$ in the neighbor image. However, the temperature of the cold population in the neighbor image is known with less precision than that of the warm water mass because it depends on $\tau_{x, y}^{c}$ and is thus susceptible to temperature offset between images. To alleviate the problem, a count of cold pixels within the window $\mathscr{W}_{x, y}$ in the neighbor image is generated for various temperature intervals, $I(\tau)=\left[\tau-0.5^{\circ} \mathrm{C}, \tau+0.5^{\circ} \mathrm{C}\right]$, such that $\tau_{x, y}^{c}-$ $0.5^{\circ} \mathrm{C} \leqslant \tau<\tau_{x, y}^{c}+0.5^{\circ} \mathrm{C}$. To speed up the algorithm, only the values $\tau=\tau_{x, y}^{c}-0.5, \tau=\tau_{x, y}^{c}$, and $\tau=\tau_{x, y}^{c}+0.5$ are checked. The value $\tau_{\max }$ which maximizes the count of valid cold pixels defines $N_{x, y}^{\text {cold }}$ :

$$
N_{x, y}^{\text {cold }}=\#\left\{(i, j)^{n} \in \mathscr{W}_{x, y} \text { such that } \tau_{x, y}^{n} \in I\left(\tau_{\text {max }}\right)\right\} .
$$

The cold water mass is said to exist when

$$
N_{x, y}^{\text {cold }}>\varepsilon_{c} \text {. }
$$

Although $\varepsilon_{c}$ was chosen here to be the same as $\varepsilon_{w}$, the two parameters are not strictly related. While the 
warm population occupies a large portion of $\mathscr{W}_{x, y}\left[(x, y)^{n}\right.$ at the center of the window is warm], the cold population may only occupy a small fraction of the window. Changing $\varepsilon_{c}$ by $\pm 50 \%$ produces a $\pm 4 \%$ in the number of pixels which are classified as clouds. However, the probability of agreement with the analyst remains unchanged.

When $(x, y)^{c}$ is between two masses of water, that is, the transition between the warm and the cold water masses, $\tau_{x, y}^{c}$ cannot be used to estimate the temperature of the cold water mass. To deal with such a case, a count of cold pixels within $\mathscr{W}_{x, y}$ in the current image with a direct correspondence with similarly cold pixels in the neighbor image is substituted for the previously defined $N_{x, y}^{\text {old }}$ if it produces a higher count.

\section{Additional Cloud Detection Using Single-Image Analysis}

The single-image cloud detection algorithm starts by adding new potentially cloudy pixels to the map, $\mathscr{C}_{p c}[\mathrm{Eq}$. (3)], which was obtained from the multiimage algorithm. Following that step, the map $\mathscr{C}_{p c}$, with the potentially cloudy pixels added by the single-image algorithm, is smoothed and segmented into connected regions. The resulting regions of potentially cloudy pixels are then analyzed for a final classification.

\section{Addition of Potential Clouds Identified by the Single-Image Algorithm}

The single-image algorithm uses two tests to flag pixels as potential clouds. The first test is a simple temperature threshold. Pixels colder than $1{ }^{\circ} \mathrm{C}$ are identified as potentially cloudy.

$$
\tau_{x, y}^{c}<1^{\circ} \mathrm{C} \Rightarrow(x, y)^{c} \in \mathscr{C}_{p c}
$$

There are two reasons for the $1^{\circ} \mathrm{C}$ threshold. First, although this does not apply at higher latitudes, in the portion of the western North Atlantic covered by the images used in this study, temperatures that are less than $1{ }^{\circ} \mathrm{C}$ are usually associated with clouds (based on the sequence of images declouded by the analyst, $94 \%$ of pixels between $0.5^{\circ} \mathrm{C}$ and $1{ }^{\circ} \mathrm{C}$ are cloudy). Second, the images available to this study were all preprocessed so that SST values lower than $0.5^{\circ} \mathrm{C}$, are set to 0 . This preprocessing threshold makes it difficult to analyze the temperature variability (next paragraph) of regions colder than $1^{\circ} \mathrm{C}$.

The second test is based on the gradient of the SST field. The algorithm identifies areas of high variability in the SST field. The following expression approximates the gradient vector ${ }^{1}$ at a location $(i, j)$.

$$
\operatorname{grad}(i, j)=\left(\begin{array}{l}
\operatorname{grad}_{x}(i, j) \\
\operatorname{grad}_{y}(i, j)
\end{array}\right)=\left(\begin{array}{c}
\tau_{i+1, j}-\tau_{i-1, j} \\
\tau_{i, j+1}-\tau_{i, j-1}
\end{array}\right)
$$

\footnotetext{
'Due to the discrete nature of the data, the step size is measured and used instead of the actual gradient.
}

Pixels are classified as potentially cloudy based on the magnitude of $\operatorname{grad}(i, j)$,

$$
\|\operatorname{grad}(x, y)\|>2.5^{\circ} \mathrm{C} \Rightarrow(x, y)^{c} \in \ell_{p c} .
$$

The $2.5^{\circ} \mathrm{C}$ threshold was determined through experimentation with several images so that when the thermal variability is caused by an SST front, only a few-pixelwide region would be classified as potentially cloudy. This threshold should work well globally because temperature steps elsewhere in the ocean are not expected to exceed those associated with the Gulf Stream. The next few processing steps lessen the impact of this threshold on the final result. A change of $\pm 20 \%$ for this threshold produces a $\mp 5 \%$ change in number of cloud-classified pixels and agreement with the analyst (see validation in the next section) varies by less than $\mp 1 \%$.

Before areas of potentially cloudy pixels are identified as individual regions, the map $\mathscr{b}_{p c}$ is processed to obtain more compact groupings of pixels and to remove thin regions of high thermal variability which are usually created by SST fronts. A pixel $(x, y)^{c}$ that belongs to $\mathscr{C}_{p c}$ is included in the new map $\ell_{p c}^{\prime}$, if a majority of the pixels within a 7 by 7 pixel window centered at $(x, y)^{c}$, $w_{x, y}$, also belongs to $\mathscr{b}_{p}$,

$$
\#\left\{(i, j)^{c} \in w_{x, y} \mid(i, j)^{c} \in \mathscr{C}_{p c}\right\} \geqslant \frac{1}{2} \# w_{x, y} \Rightarrow(x, y)^{c} \in \mathscr{C}_{p c}^{\prime} .
$$

\section{Regional Analysis}

The next step prepares for a region by region analysis. Equation (11) defines two classes of pixels: pixels that belong to $\ell_{p c}^{\prime}$ and pixels that do not belong to $\ell_{p c}^{\prime}$. Based on these two classes, the image is segmented in a partition of 4-connected regions (Duda and Hart, 1973) such that within each region, $\mathscr{R}$, all pixels belong to the same class.

Rapid changes of the SST values in a region indicate the presence of a cloud or an SST front. The goal is to differentiate high variability regions associated with clouds from those associated with SST fronts. The gradient vector field within a region and the shape of the region are analyzed to make that determination.

The gradient vector field within a region of high variability is analyzed first. This analysis relies on two observations:

1. Gradient vectors associated with an SST front have a consistent direction which is perpendicular to that of the front,

2. Gradient vectors in cloudy regions are often randomly distributed.

The coherence of the gradient vector field is measured by the ratio $\gamma_{\mathscr{S}}$ which is defined as the magnitude of the sum of gradient vectors over the sum of gradient vector magnitudes, 


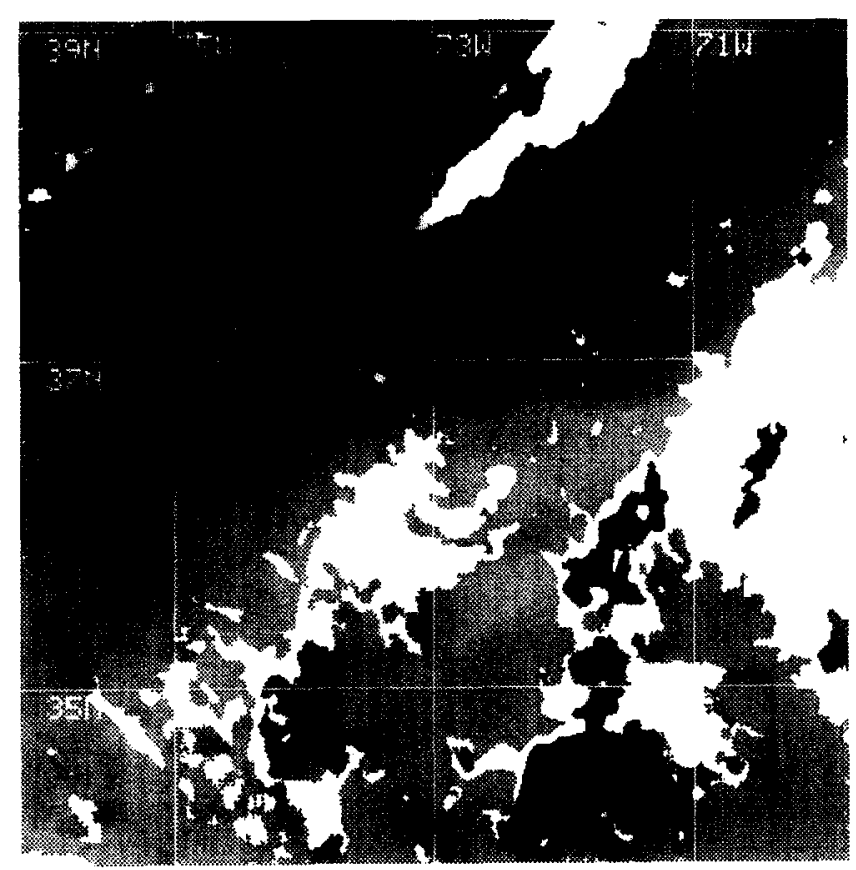

Figure 5. Image of the SST field taken on 17 April 1982 at 7:04 local time. Clouds from the single-image algorithm, as well as longitude and latitude coordinates, are overlaid in white.

$$
\gamma_{\mathscr{A}}=\frac{\left\|\sum_{(i, j) \in \mathscr{S}} \operatorname{grad}(i, j)\right\|}{\sum_{(i, j) \in \mathscr{A}}\|\operatorname{grad}(i, j)\|} .
$$

Consequently, $\gamma_{\mathscr{A}}$ tends to unity when the direction of gradient vectors is constant over the entire region $\mathscr{R}$, while, in a region of randomly distributed gradient vectors, the ratio tends to zero. After looking at several cases of SST frontal regions that were classified as potentially cloudy, the following decision rule for regions of $\mathscr{C}_{p c}^{\prime}$ pixels was chosen:

$$
\begin{aligned}
& \gamma_{: \mathscr{P}}<0.3 \Rightarrow \mathscr{R} \subset \mathscr{C}_{c} \\
& \gamma_{\mathscr{A}}>0.7 \Rightarrow \mathscr{R} \subset \mathscr{C}_{c},
\end{aligned}
$$

where $\mathscr{C}_{c}$ is the set of cloudy pixels. Changing the value of the constants $( \pm 0.1)$ does not significantly affect either the percentage of detected clouds or the rate of agreement with the analyst.

The classification of regions that are left undefined by the previous step is achieved through an analysis of their shapes: Cloud regions are often bulky while SST front regions are usually associated with elongated shapes. Although more sophisticated methods exist (Jain, 1989), analysis of the two eigenvalues, $\lambda_{1}$ and $\lambda_{2}$, of the spatial covariance matrix (Duda and Hart, 1973) is sufficient for our purpose. Tests led to the following decision rule for regions of $\mathscr{C}_{p c}^{\prime}$ pixels with $0.3 \leqslant \gamma_{\mathscr{H}} \leqslant 0.7$,

$$
\begin{aligned}
& \lambda_{1} \leqslant 6 \lambda_{2} \Rightarrow \mathscr{R} \subset \mathscr{C}_{c}, \\
& \lambda_{1}>6 \lambda_{2} \Rightarrow \mathscr{R} \subset \mathscr{C}_{c},
\end{aligned}
$$

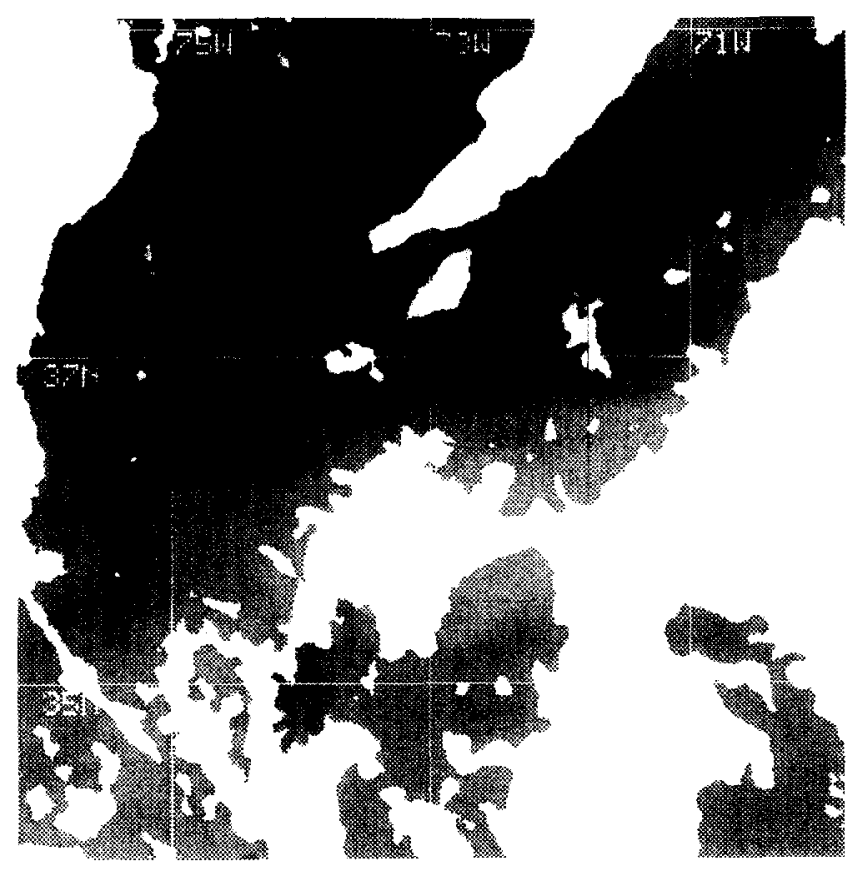

Figure 6. Image of the SST field taken on 17 April 1982 at 7:04 local time. Clouds from the integrated algorithm, as well as longitude and latitude coordinates, are overlaid in white.

where $\lambda_{1}$ refers to the large eigenvalue of the spatial covariance matrix while $\lambda_{2}$ refers to the smaller one. Values ranging from 4 to 8 for the multiplicative constant produce similar results.

Finally, clear simply connected regions that contain fewer than 400 pixels are identified as cloudy areas because within cloudy regions a small region is more likely to be cloudy than cloud-free.

In Figure 5 the clouds that are detected by the single-image analysis, without the benefit of the multiimage pass, are overlaid in white on the SST field of Figure 3. As expected, cloudy regions with low variability are not detected. Figure 6 shows the clouds detected by the integrated algorithm. By comparison with Figure 5, the integrated algorithm detects most of these cloudy regions with low variability.

\section{VALIDATION}

A gradient-based single-image cloud detection algorithm which is similar to the algorithm described in the previous section when multiimage information is unavailable was analyzed in a previous study (Kowalski et al., 1991) along with two other single-image algorithms. Of the three automatic methods, the gradient-based algorithm achieved the highest percentage of agreement with a human expert.

To validate the integrated cloud detection algorithm, its effect on a companion edge detection algorithm (Cayula and Cornillon, 1995) is analyzed. The 
Table 1. Effect of the Cloud Detection Methods on the Edge Detection Algorithm ${ }^{a}$

\begin{tabular}{lcccc}
\hline \multicolumn{1}{c}{ Method } & $N$ & $m_{\perp}$ & $\sigma_{\perp}$ & $a_{3 \perp}$ \\
\hline Single-image cloud detection & 197 & -10.84 & 18.44 & 0.77 \\
Multiimage cloud detection & 192 & -12.78 & 15.06 & -0.05 \\
Subjective analysis & 226 & -12.53 & 14.91 & -0.10 \\
\hline
\end{tabular}

${ }^{a}$ Results obtained by the analyst are given for comparison.

results are compared to those obtained by a human expert and to those obtained by the single-image edge detection algorithm applied to the same data.

The evaluation is conducted on a set of 98 SST images for which Inverted Echo Sounder (IES) data were available to determine the in situ position of the Gulf Stream northern edge along separate IES lines (Chaplin and Watts, 1984). The in situ position, denoted by $T_{15}$, is the surface projection of the $15^{\circ}$ isotherm at $200 \mathrm{~m}$. It is used as a reference to evaluate edges determined from satellite-derived imagery.

The intersection between an IES line and the Gulf Stream northern edge as determined from the satellitederived imagery defines an evaluation point. The performance analysis relies on four statistics (Cornillon and Watts, 1988):

1. The number of evaluation points $N$ that have been detected from the satellite-derived SST fields.

2. The average distance, $m_{\perp}$, between the evaluation points and the corresponding $T_{15}$ 's. A nonnull offset is expected because the $15^{\circ}$ isotherm in the vicinity of the Gulf Stream is not vertical.

The surface location of the Gulf Stream northern edge is usually shoreward of the position determined at a depth of $200 \mathrm{~m}$.

3 . The standard deviation, $\sigma_{\perp}$, of the distance between the evaluation points and the corresponding $T_{15}$ 's. This statistic measures the reliability of edges detected from SST images as estimates of $T_{15}$.

4. The skew, $a_{31}$, of the distribution. For each north to south line in an image, the southernmost edge with the proper characteristics of orientation, temperature, and location is selected as the Gulf Stream northern edge by the algorithm presented in Cayula (1993). This means that cloud-induced edges to the south of the actual Gulf Stream northern edge are the ones most likely to interfere with the selection process. As a consequence, undetected clouds tend to skew the determination of SST edges to the south.

Table 1 presents the results obtained by the multiimage edge detection algorithm (Cayula and Cornillon, 1995) when it is combined, first, with only the singleimage cloud detection algorithm, and then, with the integrated cloud detection algorithm. The same algorithm (Cayula, 1993) is used in both cases to select the Gulf Stream northern edge. Results obtained when all processing is left to a human expert are shown for comparison.

The first line of Table 1 shows that a higher value of $\sigma_{\perp}$ than that observed on the next two lines while the positive $a_{3 \perp}$ and a smaller negative mean offset indicate that the algorithm is selecting contours south of those detected by the other methods. These numbers imply that clouds left undetected by the single-image algorithm interfere with the detection of SST fronts. The integrated cloud detection algorithm corrects the problem so that the edge detection algorithm achieves results comparable to those obtained by a human expert. Although the integrated algorithm, compared to the single-image algorithm, detects a higher percentage of clouds, the number of false clouds stays small enough to not significantly affect the number $N$ of detected edges.

The preceding results are corroborated by a direct comparison of the automatically detected clouds and those subjectively detected by a human expert. The pixel by pixel comparison is conducted on a sequence of 317 images which form a superset of the development set (Holloway, 1993). Table 2 summarizes the results for the single-image, the multimage cloud detection algorithms, and a single-image expert system. The expert system (Holloway, 1993), which uses a blackboard model (Nii, 1986), improves the single-image algorithm so that fewer cloud-corrupted pixels are flagged as clear. In Table $2, P_{1}$ represents the proportion of pixels classified as cloudy by the algorithm but as clear by the expert while $P_{2}$ is the fraction of pixels classified as clear by the algorithm but as cloudy by the expert. $P_{A}$ is the fraction of pixels on which both the algorithm and

Table 2. Proportions of Pixels Classified as Cloudy by the Algorithm and Cloud-Free by the Expert $\left(P_{1}\right)$, Cloud-Free by the Algorithm and Cloudy by the Expert $\left(P_{2}\right)$, and Classified the Same by Both the Algorithm and the Expert $\left(P_{A}\right)$

\begin{tabular}{lccc}
\hline \multicolumn{1}{c}{ Method } & $P_{1}$ & $P_{2}$ & $P_{A}$ \\
\hline Single-image cloud detection & 0.03 & 0.17 & 0.80 \\
Multiimage cloud detection & 0.07 & 0.07 & 0.86 \\
Single-image expert system & 0.10 & 0.11 & 0.79 \\
\hline
\end{tabular}


the expert agree: $P_{A}=1-\left(P_{1}+P_{2}\right)$. The expert identified $44 \%$ of the pixels as cloudy and $56 \%$ as cloud-free.

The results of Table 2 should not be considered as an absolute measure of one algorithm performance as the subjective classification by the expert is also prone to errors. However, those results offer some useful insights. The multiimage algorithm achieves a sharp decrease in the value of $P_{2}$ (a difference of $10 \%$ ) when compared to the single-image algorithm. This indicates that the multiimage algorithm misses far fewer clouds than the single-image algorithm. Furthermore, there is only a small increase in the number of false clouds as indicated by the $4 \%$ change in the value of $P_{1}$. In comparison, the expert system succeeds in reducing the probability of missing clouds, $P_{2}$, by $6 \%$ at the cost of increasing the probability of detecting false cloud, $P_{1}$, by $7 \%$. Overall, the level of agreement with the expert, $P_{A}$, goes from $80 \%$ for the single-image algorithm to $86 \%$ for the multiimage algorithm. The value, $P_{A}$, indicates that the multi-image algorithm, by using the information found in a sequence of images, achieves a more reliable classification than either the single-image algorithm or the expert system.

\section{SUMMARY}

The goal of the integrated cloud detection algorithm was to detect clouds associated with cooler temperatures or an increased variability of the satellite-derived SST fields. Relatively cooler regions were identified by a comparison of the SST fields in a sequence of images. Regions of high variability were detected by thresholding the gradient image. Further analysis of the SST fields made it possible to differentiate relatively cooler temperatures and increased variability associated with SST frontal activity from that caused by the presence of clouds. Although the single-image cloud detection was previously shown to work well, the additional multiimage analysis improves the overall results. Such an integrated approach is necessary for the reliable detection of fronts. Despite generally good results, one should be aware of a few potential problems which have not been addressed. If a sequence of images is very cloudy such that clear data are not available within the time interval under study, even obvious cloudy areas may not be flagged. Oceanographic features must also be persistent, the algorithm may fail when strong wind events entrain colder water at the surface. On the other hand, stationary clouds may fool the algorithm. Finally, the integrated algorithm does not take into account warm clouds. As a result, a clear region in one image may be misclassified as cloudy when it is compared to an area corrupted by warm clouds in a neighbor image. These problems may be alleviated by analyzing longer image sequences to determine acceptable SST values and by using additional sources of information. As an alternative, this cloud detection algorithm could be included in a multichannel algorithm.

This research was performed with support from the National Aeronautics and Space Administration through Grant No. NAGW-3009, the Jet Propulsion Laboratory as part of the NSCAT Project through Grant No. 957627, and the Global Ocean Monitoring and Prediction (GOMAP) project via the Strategic Environmental Research and Development Program (SERDP). Salary support for P. Cornillon was provided by the State of Rhode Island and Providence Plantations. The satellite data processing software used to preprocess the imagery was developed by R. Evans, O. Brown, J. Brown, and A. Li of the University of Miami. Their continued support is greatly appreciated.

\section{REFERENCES}

Cayula, J.-F. (1993), Automatic front detection using a sequence of SST images, Ph.D. thesis, Department of Electrical Engineering, University of Rhode Island.

Cayula, J.-F., and Cornillon, P. (1995), Multi-image edge detection for SST images, J. Atmos. Ocean. Technol. 12:821829.

Cornillon, P., and Stramma, L. (1985), The distribution of diurnal sea surface warming events in the western Sargasso Sea, J. Geophys. Res. 90:11,811-11,815.

Cornillon, P., and Watts, D. R. (1987), Satellite thermal infrared and inverted echo sounder determination of the Gulf Stream northern edge, J. Atmos. Ocean. Technol. 4:712-723.

Cornillon, P., Gilman, C., Stramma, L., Brown, O., Evans, R., and Brown, J. (1987), Processing and analysis of large volume of satellite-derived thermal infrared data, J. Geophys. Res. 92:12,993-13,002.

Chaplin, G., and Watts, D. R. (1984), Inverted echo sounder development, Ocean '84 Conf. Rec. 1:249-253.

Cushman-Roisin, B. (1994), Introduction to Geophysical Fluid Dynamics, Prentice Hall, Englewood Cliffs, NJ, pp. 5-12.

Derrien, M., Legleau, H., Lavanant, L., Harang, L., Noyalet, A., and Pochic, D. (1990), Détection automatique des nuages avec l'imageur AVHRR du satellite défilant NOAA11. Note de travail EERM 4, Établissement d'Études et de Recherches Météorologiques, Direction de la Météorologie Nationale, Ministère de l'Équipement, des Transports et de la Mer, Paris.

Duda, R., and Hart, P. (1973) Pattern Recognition and Scene Analysis, Wiley, New York, pp. 284-287, 362-367.

Gallegos, S., Hawkins, J., and Cheng, C. F. (1993), A new automated method of cloud masking for advanced very high resolution radiometer full-resolution data over the ocean. J. Geophys. Res. 98:8505-8516.

Holloway, D. (1993), Blackboard system for knowledge-based interpretation of clouds in Advanced Very High Resolution Radiometer (AVHRR) imagery, Ph.D. thesis, Department of Computer Science and Experimental Statistics, University of Rhode Island

Jain, A. K. (1989), Fundamentals of Digital Image Processing, Prentice Hall, Englewood Cliffs, NJ, pp. 384-394.

Kowalski, J., Cornillon, P., Cayula, J.-F., Gallagher, J., Holloway, D., and Slawinski, O. (1991), Approaches to cloud 
detection in AVHRR images, in Proceedings: Automated Interpretation of Oceanographic Satellite Images Workshop (Matthew Lybanon, Ed.), NOARL SP 001:321:91, Stennis Space Center, MS, pp. 169-176.

McClain, E. P., Pichel, W. G., Walton, C. C., Ahmad, Z., and Sutton, J. (1983), Multi-channel improvements to satellitederived global sea surface temperatures, Adv. Space Res. 2:43-47.

Nii, H. P. (1986), Blackboard systems, Al Mag. 7(3):40-53 and $7(4): 82-107$
Podestá, G., Browder, J., and Hoey, J. (1993), Exploring the association between swordfish catch rates and thermal fronts on U.S. longline grounds in the western North Atlantic, Continental Shelf Res. 13:253-277.

Schwalb, A. (1978), The TIROS-N/NOAA-G satellite series, NOAA Tech. Memo. NESS 95, National Environmental Satellite Service, NOAA, U.S. Dept. of Commerce, Washington, DC.

Stewart, R. (1985), Methods of Satellite Oceanography, University of California Press, Los Angeles, pp. 128-152. 